\title{
Viñetas de vida: una propuesta de Aprendizaje-Servicio a través del cómic ${ }^{1}$
}

\author{
GUADALUPE ESPINOSA-GUERRI \\ Universitat de València \\ guadalupe.espinosa@uv.es
}

\begin{abstract}
Resumen: El enfoque Aprendizaje-Servicio consiste en aprender ejecutando tareas que repercutan positivamente en la comunidad. A través de este enfoque proponemos una unidad didáctica para los niveles C1-C2. La unidad se divide en tres partes: en la primera se introduce el tema mediante tareas de comprensión e interacción oral. En la segunda, se trabaja la comprensión lectora a partir del cómic Viñetas de vida, cuyo objetivo es sensibilizar a la población de la necesidad de la cooperación internacional. Finalmente, en la tercera parte, se realiza la tarea final de expresión escrita que consiste en la de redacción de un informe en que se exponen datos relevantes sobre la cooperación y se propone un plan de acción viable para el grupo. Con esta unidad didáctica se pretende, pues, involucrar al alumnado en el aprendizaje de la lengua mientras actúan sobre necesidades reales del entorno.
\end{abstract}

Palabras clave: enfoque Aprendizaje-Servicio, enfoque por tareas-proyectos, aprendizaje significativo, aprendizaje basado en la acción, cómic, cooperación al desarrollo internacional.

\section{Viñetas de vida: a proposal of Service-Learning through the comic}

Abstract: Service-Learning approach consists on learning by doing tasks that have a positive effect in the community. Through this approach we propose a teaching unit for C1-C2 levels. The unit is divided in three parts: in the first one we introduce the theme with the oral comprehension and interaction activities. In the second one, we work on reading comprehension of Viñetas de vida, a comic whose objective is to make the people aware of the importance of international cooperation. Finally, in the third part, students do the final task that consists on writing a report in with students inform about cooperation politics and proposing a viable action plan for the group. With this teaching unit we try to involve students in the language learning while acting on real needs of the environment.

Key words: Service-Learning approach, task/project-based approach, meaningful learning, action-oriented approach, comic, international cooperation and development.

\section{Introducción}

El enfoque Aprendizaje-Servicio (ApS) ha sido ampliamente utilizado en los niveles de Primaria, Secundaria y en los niveles universitarios, sin embargo, tiene poca tradición

\footnotetext{
${ }^{1}$ Este artículo ha sido posible gracias al proyecto de investigación FFI2016-77842-P, Unidades discursivas para una descripción sistemática de los marcadores del discurso en español (UDEMADIS), financiado por el MINECO, la AEI y los fondos FEDER.
} 
en la enseñanza de español como lengua extranjera (E/LE). A través de esta propuesta didáctica mostramos una aplicación de este enfoque para los niveles avanzados de español, a la vez que se analizan las ventajas y posibles problemas del mismo.

\section{Definición y delimitación conceptual del enfoque ApS}

Se trata de una propuesta educativa que «combina procesos de aprendizaje y de servicio a la comunidad en un único proyecto bien articulado en el que los participantes aprenden a la vez que trabajan en necesidades reales del entorno con la finalidad de mejorarlo» (citado en Puig et al. 2007:53). La característica principal que identifica este enfoque y lo separa de otras prácticas de educación experiencial es, por un lado, el equilibrio entre los contenidos curriculares y los objetivos didácticos; y, por otro, los resultados del servicio a la comunidad por otro (Sigmon 1999). Un ejemplo de proyecto de ApS realizado con alumnos de más de 18 años es la participación de estudiantes de Administración y Dirección de Empresas que diseñan y dinamizan un taller de expresión oral con alumnos de Secundaria. Los aprendizajes adquiridos en este proyecto son: autoconfianza, empatía, control del estrés, habilidades comunicativas, liderazgo y conducción de grupos (Fundación Zerbikas 2013).

\section{Principios y metodología de Aprendizaje-Servicio}

Subyace a este enfoque el principio de aprender haciendo, es decir, se trata de un enfoque orientado a la acción, en sintonía con el enfoque adoptado por el Marco Común Europeo de Referencia para las lenguas. Este se centra en la acción en tanto que considera a los usuarios y aprendientes de lenguas como agentes sociales (Instituto Cervantes 2002). Se trata de un tipo de enfoque por tareas-proyectos donde el proyecto desarrollado tiene un marcado componente social vinculado siempre con el entorno y la realidad social presente. La planificación ha de ser sistemática y seguir unos pasos básicos: 1) diagnóstico de las necesidades sociales del entorno; 2) selección del proyecto según los contenidos curriculares, los objetivos didácticos y la problemática social sobre la que se quiera actuar; 3) sensibilización del alumnado y profesorado; 4) diseño y organización del plan de acción estableciendo las fases y actuaciones que se llevarán a cabo para alcanzar los objetivos planteados; 5) ejecución de la propuesta; y 6) evaluación de los resultados. La evaluación tiene un papel relevante en ApS porque sirve para comprobar el alcance del servicio, dotar de sentido pleno al proyecto y mejorar las futuras acciones (Puig et al. 2007). En la fase de selección del proyecto es imprescindible tener en cuenta la viabilidad de la propuesta, puesto que una parte fundamental del enfoque es la realización del plan de acción.

\section{Beneficios y problemas}

El enfoque permite conectar teoría-práctica, aula-entorno, aprendizaje-compromiso social (Rodríguez 2014) en tareas altamente contextualizadas. Esa vinculación directa con la comunidad dota de significatividad al aprendizaje, porque se actúa sobre demandas concretas y reales de la sociedad (2014). Favorece la implicación activa en el proceso de aprendizaje tanto de alumnos como de profesores, así como el trabajo cooperativo entre iguales y con personas adultas externas al centro. Hay una mayor presencia de contenidos prácticos y estos se adaptan al contexto social del entorno (2014). Permite cohesio- 
nar diversos contenidos curriculares de forma coherente dentro del marco de un proyecto solidario.

No obstante, no es fácil ejecutar este enfoque de forma adecuada, pues los proyectos son complejos y existe el riesgo de plantear un plan de acción demasiado pretencioso cuyos objetivos sean difíciles de alcanzar. La conjunción de ambas partes debe potenciar el efecto que tendrían por separado: el aprendizaje mejora el servicio aportando contenidos de calidad al proyecto y el servicio mejora el aprendizaje porque lo dota de realidad y permite obtener conocimientos y competencias nuevas (Lucas y Martínez-Odría 2012). Este enfoque, como tipo específico de trabajo por proyectos, se enmarca dentro del aprendizaje por descubrimiento. Ferrero (2017) advierte que, para aplicar el aprendizaje por descubrimiento, es necesario que los alumnos tengan una base de conocimientos previos sobre la cual construir conocimiento nuevo y, en definitiva, para poder sacar provecho del enfoque. Por esta razón, no recomienda aplicar el aprendizaje por descubrimientos sobre contenidos totalmente nuevos ni con alumnos de Infantil, Primaria o los Primeros ciclos de la ESO, porque el objetivo en estos cursos es que los alumnos adquieran conocimientos y habilidades básicas. Por tanto, en E/LE, los niveles en los que los alumnos pueden obtener un mayor rendimiento a través del ApS son los alumnos de niveles más avanzados, $\mathrm{C} 1$ y $\mathrm{C} 2$, o quizás $\mathrm{B} 2$ en algunos casos. Teniendo en cuenta estas advertencias, Ferrero apunta también la utilidad del aprendizaje por descubrimiento para favorecer la aplicación y adquisición de conocimientos complejos y abiertos.

\section{Unidad didáctica Viñetas de vida}

\subsection{Nivel, destrezas y contenidos}

La propuesta consiste en una unidad didáctica que gira en torno al cómic Viñetas de Vida (Cebrián et al. 2014) y al tema de la cooperación internacional al desarrollo. Está destinada a los niveles superiores de lengua, C1-C2 y se trabajarán a lo largo de las actividades todas las destrezas (comprensión, expresión e interacción oral y escrita). Debido a que el tema central es de cierta complejidad, se recomienda su utilización con alumnos mayores de dieciocho años.

Los contenidos tratados son, a nivel gramatical, el uso del condicional para formular propuestas y sugerencias, el pretérito imperfecto con valor de futuro, el presente con valor de pasado, partículas discursivas para ordenar y argumentar y algunos mecanismos de impersonalidad. A nivel funcional, los alumnos aprenderán a dar y pedir la opinión cortésmente, hacer preguntas y sugerencias, a argumentar y explicar. Los contenidos léxicos son el vocabulario relacionado con la solidaridad, la desigualdad social, la cooperación al desarrollo y los valores personales, así como el metalenguaje del cómic (viñeta, bocadillo, onomatopeya, etc.). A nivel pragmático se introducen algunos indicadores léxico-semánticos de la ironía, concretamente, las contradicciones, generalizaciones y mentiras irónicas; y algunas fórmulas de cortesía. También se trabajan habilidades interculturales, concretamente, el uso de conocimientos para identificar diferencias culturales relacionadas con la historia de diferentes países. Además, se trabajarán dos géneros textuales: el cómic desde la comprensión lectora y el informe desde la expresión escrita. 


\subsection{Estructura de la unidad}

La unidad se divide en tres partes, más la evaluación final de la unidad y del proyecto. En la primera parte introducimos a los alumnos en el tema de la desigualdad social y la cooperación al desarrollo para activar los conocimientos previos, presentar el vocabulario básico de la unidad y obtener algunos datos para la evaluación inicial. En la segunda parte se trabajará el tema de la cooperación al desarrollo y los contenidos lingüísticos a través de la comprensión y comentario de dos lecturas, Ondas en el río, de Cristina Durán y Miguel Ángel Giner Bou; y Los niños sin espejo, de David Rubín. En la tercera parte se realizará la tarea final que consistirá en la elaboración de un informe en el cual los alumnos informarán sobre las políticas de cooperación internacional y propondrán un plan de acción para el grupo o el centro educativo. Dicho plan de acción consistirá en una campaña divulgativa sobre la cooperación al desarrollo. Se debatirán las diferentes propuestas y se votarán posteriormente. Tras la selección de una de ellas, se llevará a la práctica. Se inicia en este punto la fase de servicio, aunque el aprendizaje continúa hasta la evaluación final del proyecto.

\subsection{Primera parte: nos ponemos en marcha}

La primera actividad que proponemos consiste en reflexionar y comentar con el grupo las siguientes cuestiones:

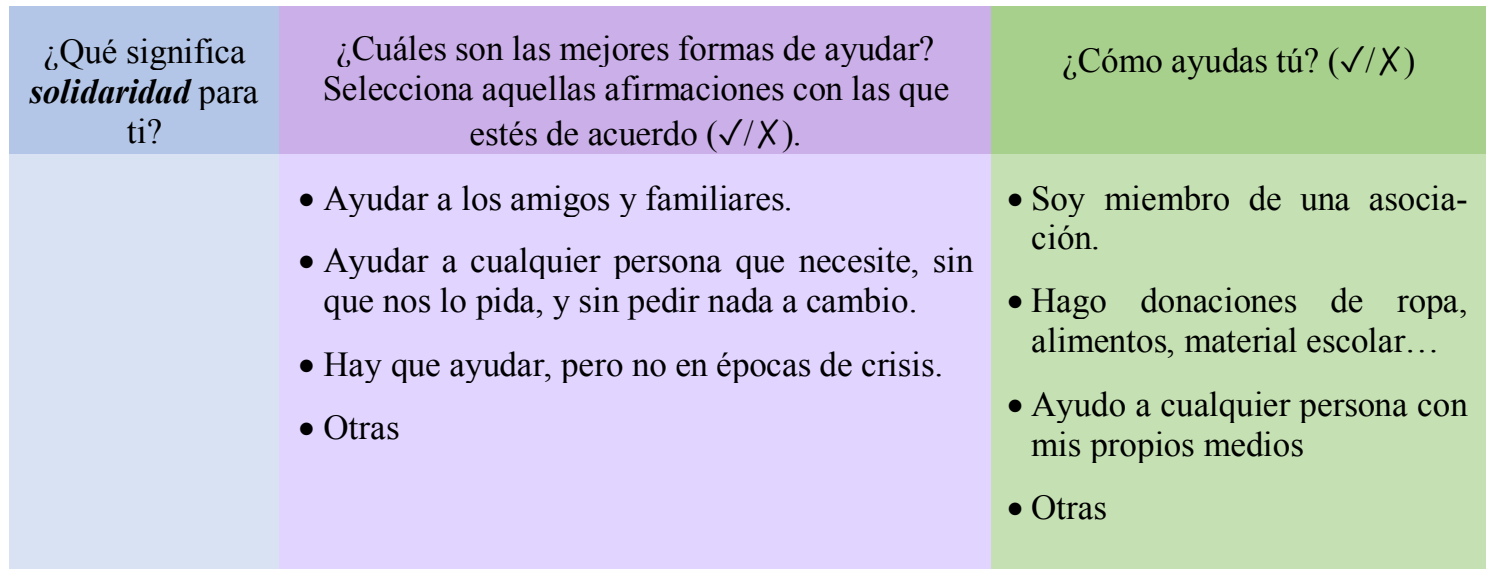

Tabla 1. Preguntas de reflexión inicial

En primer lugar, dejamos un tiempo para reflexionar individualmente y buscar las palabras nuevas en el diccionario. Luego comentamos las respuestas en pequeños grupos o todos juntos. Los objetivos son, introducir el tema de la unidad, activar conocimientos previos y presentar algunas de las palabras básicas, como solidaridad o cooperación al desarrollo internacional. Asimismo, esta tarea sirve para sensibilizar al alumnado y realizar una evaluación inicial sobre sus conocimientos del tema (ver $\S 4$, p. 9).

En la segunda actividad proponemos el visionado de un falso reality, llamado 3 de 10: El reality de la cooperación ${ }^{2}$. Podemos realizar un primer visionado del vídeo antes de plantear las preguntas de comprensión oral y presentar las preguntas antes del segundo

\footnotetext{
${ }^{2}$ Enlace del reality: https://www.youtube.com/watch?v=SjJzvNMsNuE
} 
visionado: ¿Por qué el reality se llama 3de10? Es un falso reality, ¿en qué lo notas? (en el decorado, en el aspecto de la presentadora, en la forma de hablar, en el tema, en la música...) ¿Cómo es el tono del programa? (serio, humorístico, paródico, irónico...) ¿Cuál crees que es el objetivo del reality? (hacer reír, entretener al espectador, conmover, impactar, hacer una crítica social...) ¿Qué piensas después de ver el reality? ¿Descartarías a alguno de los participantes del reality?

A través de estas cuestiones podemos extraer más vocabulario del tema y trabajar los indicadores léxico-semánticos de la ironía que la presentadora emplea: contradicciones, generalizaciones y mentiras irónicas; recursos utilizados con el objetico de impactar al espectador, conmoverlo y hacer una dura crítica a los resortes en las políticas de cooperación al desarrollo. Utilizando estas mismas preguntas de comprensión lectora proponemos simultáneamente una tarea de interacción oral en la que los alumnos aprenden a dar y pedir la opinión de forma cortés. Para ello les ofrecemos las siguientes estructuras corteses: a) para dar la opinión: A mi entender/parecer, se trata de un falso reality porque...; A mi juicio, se llama 3 de10 porque...; A mi me da la sensación/impresión que el objetivo del reality es...; b) para pedir la opinión: ¿Qué piensas/opinas de lo que dice la presentadora?; ¿Qué te parece lo que acabo de decir?; ¿Dirías que el video consigue su objetivo?; A tu entender/parecer, ¿qué es lo que pretende transmitir el vídeo?

\subsection{Segunda parte: leemos Viñetas de vida}

En la segunda parte, vamos a profundizar en el tema a través de dos lecturas del cómic y trabajaremos varios contenidos. La primera lectura, Ondas en el río nos servirá para tratar los contenidos de gramática, pragmática y léxico; con la segunda, Los niños sin espejo introduciremos habilidades interculturales y recursos del lenguaje visual del género del cómic.

La tercera actividad consiste en crear y participar en un club de lectura virtual. Esta actividad se realiza tras una primera lectura individual de las dos historias. El grupo de lectura se puede crear a través de blogger, redes sociales como Facebook, Twitter o Instagram, o en espacios más especializados como Goodreads. Una vez creado el club, animamos a los alumnos a entrar y hablar sobre el argumento, la finalidad de la obra, las ilustraciones, el estilo gráfico o el lenguaje con la ayuda de las estructuras de dar y pedir la opinión trabajadas en la actividad 1.

Aprovechamos esta actividad para introducir el metalenguaje del género: viñeta, tira, historieta, bocadillo, onomatopeya, novela gráfica, cómic, tebeo, dibujante de cómic, ilustrador/a, guionista. Para ello pedimos a los estudiantes que introduzcan estas palabras en el hueco correspondiente de la imagen 1: 


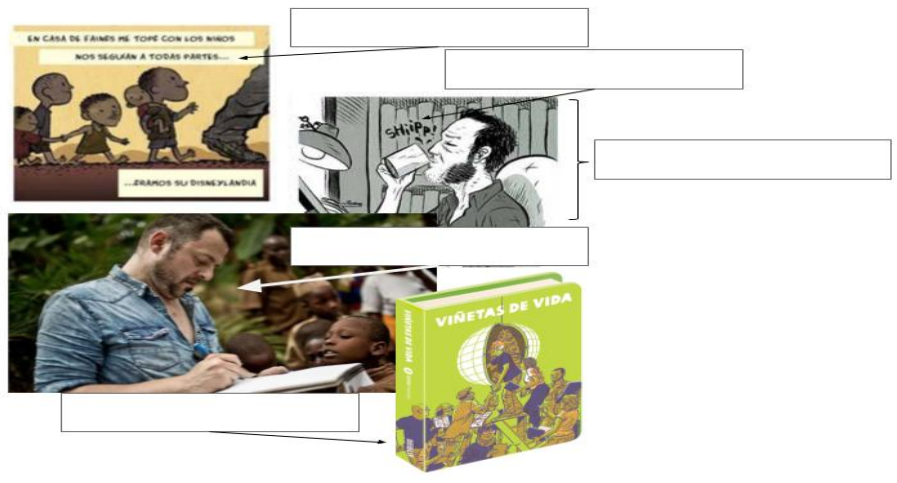

Imagen 1. Actividad metalenguaje del cómic

No todas las palabras de la lista están en la imagen, de modo que, una vez identificadas aquellas que sí tienen imagen, se pueden comentar aquellas que no la tienen y que presentan alguna dificultad. De hecho, hemos escogido términos con significados muy próximos, como dibujante de cómic e ilustrador, o novela gráfica, cómic, tebeo e historie$t a$, para que los alumnos busquen y discutan las diferencias entre ellos.

\subsubsection{Ondas en el río}

Ondas en el río, de Cristina Durán y Miguel Ángel Giner Bou habla sobre el viaje de estos autores a Nicaragua, donde conocen proyectos como el de Fundación Entre Mujeres, una cooperativa de mujeres agrícolas que se organizan alrededor de la producción propia del café. La historia cuenta las vivencias de estos autores en distintas poblaciones y la repercusión de la cooperación internacional sobre la vida de las mujeres y familias nicaragüenses.

La cuarta actividad trata sobre el uso de los tiempos dislocados, concretamente, el pretérito imperfecto con valor de futuro y el presente con valor de pasado. En la segunda viñeta de la historia, uno de los personajes dice: “¿dónde me has dicho que ibas?”. Llamamos la atención sobre esta oración y plantemos una serie de cuestiones para reflexionar sobre este uso: ¿Hace referencia a una acción presente, pasado o futura? ¿Qué tiempos verbales utiliza? ¿Crees que significa lo mismo que dónde me has dicho que irás? Y les pedimos que localicen más usos como este en la historia.

Lo mismo hacemos más adelante con el uso del presente con valor de pasado, primero llamamos la atención sobre la oración "nada más llegar nos llama la atención el colorido" (situada en la cuarta viñeta) y después planteamos las mismas preguntas. El objetivo es que los alumnos descubran, guiados por las preguntas, que algunas formas verbales que ya conocen, como el pretérito imperfecto o el presente de indicativo, a veces se pueden utilizar para expresar otros valores temporales y tienen significados pragmáticos distintos: el pretérito imperfecto con valor de futuro sirve para pedir de nuevo una información que ya se ha recibido previamente pero que, por alguna razón, no se recuerda. Por su parte, el presente de indicativo con valor de pasado sirve para acercar simbólicamente una acción pasada al tiempo de la enunciación, o para extender una acción pasada hasta el tiempo de la enunciación. 
La quinta actividad consiste en la confección de un glosario colectivo con términos específicos del tema de la cooperación internacional al desarrollo y la desigualdad social. Primero pedimos a cada alumno que busque cinco palabras en la lectura para, después, ponerlas en común y elaborar entre todos el glosario. Algunas de las palabras que pueden encontrar son infraestructura, cooperativa, derechos laborales, recursos naturales o red de apoyo.

\subsubsection{Los niños sin espejo}

La historia de David Rubín, Los niños sin espejo, está dedicada y dirigida a su hija. En ella denuncia las injusticias que presencia en Burundi y que su hija nunca llegará a vivir por haber nacido en un país desarrollado. Rubín escribe la obra con el objetivo de que su hija y todos nosotros conozcamos la vida de los niños de Burundi y que no los olvidemos.

Debido a las comparaciones que Rubín realiza entre Burundi y España, esta historia resulta útil para trabajar habilidades interculturales. Por ello, en la cuarta actividad se plantean dos bloques de preguntas. El primero: el autor explica el contexto político actual de Burundi y lo compara con un periodo del pasado de España. ¿A qué periodo se refiere?, ¿cuáles son las semejanzas entre el Burundi actual y el pasado de España? ¿Por qué crees que hace esta comparación? A través de estas cuestiones podemos trabajar contenidos de la historia de España y del periodo de la dictadura franquista con el que se compara la situación de Burundi en la actualidad, para buscar los puntos en común y las diferencias. Asimismo, motivamos una reflexión sobre cuál podría ser la finalidad del autor al utilizar el recurso de la comparación. Se trata de ayudar a interpretar el texto y a obtener información más allá de significado literal de las palabras. Lo mismo pretendemos a través del segundo bloque de preguntas: al final de la historia, David Rubín compara el futuro de su hija neonata con el de los niños de Burundi, ¿cuáles son las diferencias que señala? ¿Cuál es el objetivo de esta comparación?

En la sexta actividad llamaremos la atención sobre los recursos visuales del género del cómic que utiliza el autor a través de las siguientes cuestiones: 1) Observa los colores que utiliza el autor a lo largo de la historia. ¿Crees que pueden tener algún significado? Rubín emplea tonos oscuros y rojos de forma marcada, y lo hace cuando quiere trasmitir violencia, oscuridad o miedo. 2) La mayoría de las viñetas del cómic presentan planos muy cercanos, ¿qué efecto tiene este recurso en el lector? La cercanía de los planos es un recurso muy utilizado en la obra para acercarnos a la situación de un país tan lejano como Burundi o para generar mayor empatía. 3) La actitud del autor cambia en la última página de la historia, ¿qué diferencias ves? El tono general de la historia es crítico, pesimista, triste; pero al final se percibe un tono más esperanzado que se traduce en un uso de colores más claros y de escenas más luminosas. Quizás el objetivo sea plasmar una mirada optimista hacia el futuro de Burundi, de sus niños y también de España.

\subsection{Tercera parte: Planificamos y actuamos}

Llegamos a la tercera y última parte de la unidad, en la cual se realiza la tarea final de redacción del informe, junto con la puesta en común de las propuestas, la elección del proyecto y la planificación de la actuación para su puesta en práctica. 
La séptima actividad consiste en la elaboración del informe individualmente. En primer lugar, pedimos a los alumnos que investiguen sobre las políticas de cooperación al desarrollo de España o del país en el cual se esté realizando el curso de español. Deberán recoger la información más importante ${ }^{3} \mathrm{y}$, a partir de ella, elaborar una propuesta de plan de acción para el grupo o centro educativo. La elección del proyecto se hará teniendo en cuenta tres criterios fundamentales: 1) viabilidad real del proyecto, según el número de participantes, personas externas o instituciones que puedan colaborar, materiales, tiempo y recursos de los que se dispone, etc., 2) capacidad de impacto sobre el problema, se ha de buscar el plan de acción que mayor repercusión y mayores probabilidades de éxito tenga, y 3 ) originalidad, cuanto más interesante y diferente sea el proyecto, más posibilidad de impacto tendrá y permitirá disfrutar más al grupo durante su realización.

Proponemos como condición básica para el diseño de las propuestas que todas tengan como objetivo elaborar una campaña para informar sobre qué es la cooperación al desarrollo y por qué es necesaria. De esta manera, limitamos la variedad de propuestas que los alumnos puedan realizar a la vez que establecemos un objetivo más concreto y realista, evitando así que se propongan acciones demasiado pretenciosas. El profesor también puede establecer previamente cuáles van a ser los destinatarios de la campaña: los alumnos y profesores del centro, los vecinos del barrio, las escuelas de español de la ciudad, etc. Asimismo, podemos proporcionar algunos modelos de campañas que se podrían llevar a cabo: grabación de un vídeo para proyectar en el centro educativo y sensibilizar a los estudiantes y alumnos sobre la importancia de las políticas de cooperación al desarrollo; diseño de un póster con una infografía que informe sobre el tema para difundirlo por los centros de enseñanza de español o por los institutos de educación secundaria.

Como ayuda para la redacción del informe les facilitamos el siguiente esquema: 1) exposición de los datos más relevantes sobre la cooperación al desarrollo, la pobreza y la desigualdad; 2) desarrollo de la propuesta de plan de acción para el grupo o centro educativo explicitando el objetivo del plan de acción, el lugar donde se realizará y las fases para la realización del plan y recursos necesarios; 3) conclusión con las razones principales por las que la campaña es necesaria y adecuada para el objetivo planteado. Para ello proporcionamos una serie de consejos estilísticos y recursos lingüísticos que resumimos en la siguiente tabla:

\begin{tabular}{|l|l|}
\hline \multicolumn{1}{|c|}{ Consejos estilísticos } & \multicolumn{1}{c|}{ Recursos lingüísticos } \\
\hline $\begin{array}{l}\text { El objetivo es informativo, por lo que } \\
\text { debes evitar dar tu opinión. No utili- } \\
\text { ces expresiones como creo que, pien- } \\
\text { so que, a mi parecer... }\end{array}$ & $\begin{array}{l}\text { Mecanismos de impersonalidad: } \\
\text { paña a la cooperación se han reducido en un } \\
x \% .\end{array}$ \\
\hline $\begin{array}{l}\text { Utiliza un lenguaje claro y sencillo: } \\
\text { evita la adjetivación innecesaria y las }\end{array}$ & Tiempos verbales: \\
\hline
\end{tabular}

\footnotetext{
${ }^{3}$ En el caso de España, se puede encontrar información sobre la cooperación al desarrollo en la página del Ministerio de Asuntos Exteriores:

http://www.exteriores.gob.es/Portal/es/PoliticaExteriorCooperacion/CooperacionAlDesarrollo/Paginas/Ini cio.aspx
} 


\begin{tabular}{|l|l|}
\hline $\begin{array}{l}\text { oraciones subordinadas excesivamen- } \\
\text { te largas. }\end{array}$ & $\begin{array}{l}\text { - Presente de indicativo: informar, ofrecer datos } \\
\text { - Pretérito perfecto: exponer hechos recientes } \\
\text { - Futuro simple: proponer actuaciones }\end{array}$ \\
\hline $\begin{array}{l}\text { Aporta datos objetivos y cita las fuen- } \\
\text { tes de donde extraigas la información. }\end{array}$ & $\begin{array}{l}\text { Ordenación del discurso: primeramente, en pri- } \\
\text { mer/segundo/tercer lugar, en conclusión, para con- } \\
\text { cluir, etc. }\end{array}$ \\
\hline $\begin{array}{l}\text { Expón de forma clara el objetivo de la } \\
\text { propuesta. }\end{array}$ & $\begin{array}{l}\text { Argumentación: por consiguiente, por el contrario, } \\
\text { contrariamente, no obstante, debido a, gracias a, a } \\
\text { causa de, etc. }\end{array}$ \\
\hline
\end{tabular}

Tabla 2. Resumen de consejos estilísticos y recursos lingüísticos para el informe

Tras la elaboración individual del informe, se ponen en común todas las propuestas. En esta parte puede ser interesante emplear las estructuras para dar y pedir la opinión cortésmente que hemos trabajado en la segunda actividad (\$3.3, pp. 4-5). Una vez elegido el proyecto, el siguiente paso es dividir las tareas entre los miembros, fijar un calendario con las fases de actuación y pensar en alternativas a las que poder recurrir en caso de que surja algún problema en alguna de las fases. La buena planificación del proyecto y la previsión de posibles problemas es una parte fundamental de la tarea. En esta parte es importante establecer cómo se llevará a cabo la evaluación inicial de la situación antes de la realización de la campaña y cómo se evaluarán los resultados finales del plan. Una opción posible es que los alumnos diseñen una encuesta sobre el conocimiento que tienen los futuros receptores de la campaña antes de la puesta en marcha del proyecto y otra tras su realización para comprobar el grado de incidencia de la campaña a nivel informativo y a nivel actitudinal. Una vez tengamos claro qué hacer, cómo actuar y cómo evaluar los resultados de la campaña, solo queda ponernos en marcha.

\section{Evaluación de la unidad y del proyecto}

La evaluación inicial de los conocimientos previos de los alumnos se realiza a través de la primera actividad $(\S 3.3$, p. 4). Para ello se puede utilizar una plantilla de observación en que el profesor puntúa del 0 al $5(0=$ nada, $5=$ mucho $)$ los siguientes aspectos: a) ¿Conocían los conceptos clave previamente?; b) ¿Han tenido dificultades para entender los conceptos clave?; c) ¿Tienen una opinión formada sobre el tema?; d) ¿Muestran interés por saber más sobre tema?

Una vez finalizada la tercera parte de la unidad, y previamente a la puesta en marcha del plan de acción, los alumnos deberán realizar una autoevaluación a través de un formulario en línea ${ }^{4}$. El formulario tiene tres partes: la primera para recopilar los datos de los alumnos (nombre, apellidos y correo electrónico), la segunda con preguntas para autoevaluar el aprendizaje, y la tercera para evaluar la unidad. El formato de las preguntas es

\footnotetext{
${ }^{4}$ Se puede acceder al formulario en línea a través de esta dirección: https://docs.google.com/forms/d/e/1FAIpQLSelMowl3LBAFtgfEwDPbcG0MYF5TwWts1n6_PnT8gZHBIC2Q/viewform
} 
variado: de respuesta breve, de respuesta larga y preguntas escalares para puntuar del 0 al 5 .

Para evaluar el proyecto proponemos elaborar un diario visual conjunto que permita hacer un seguimiento tanto del proceso como del resultado final. Asignamos la narración de un día o de una fase del proyecto a cada alumno, dependiendo de lo numeroso que sea el grupo. Si se realiza por días, el alumno encargado de narrar esa jornada deberá realizar fotos durante la sesión de trabajo y seleccionar una que sea representativa de las tareas realizadas para añadirla al diario junto con una pequeña descripción y una valoración personal. En la valoración es importante que se comenten los aspectos positivos y negativos 5 .

\section{Conclusiones}

A través la unidad didáctica Viñetas de vida, hemos propuesto la aplicación del Aprendizaje-Servicio para alumnos adultos de niveles avanzados (C1-C2). Consideramos que no es una propuesta adecuada para los niveles inferiores o para alumnos demasiado jóvenes debido a que se trata de un tipo de enfoque por proyectos basado en el aprendizaje por descubrimientos (ver Ferrero 2017) y debido a la complejidad del tema y los contenidos. La dificultad del enfoque adoptado se ve compensada por su capacidad para adquirir y aplicar contenidos abiertos y complejos. Permite integrar de una forma coherente varios contenidos curriculares que, a menudo, pueden resultar difíciles de cohesionar y que se suelen impartir de forma parcelada. Aporta significatividad al aprendizaje porque las tareas están vinculadas directamente con el entorno cercano del alumno. A lo largo de la unidad se trabajan contenidos gramaticales, funcionales, léxicos, pragmáticos e interculturales, y dos géneros textuales: el cómic a nivel receptivo, y el informe a nivel productivo. Se desarrollan todas las destrezas comunicativas y se incluyen actividades de trabajo individual y de trabajo cooperativo. Como rasgo distintivo con respecto a otras propuestas de ApS, el plan de acción que se realiza la proponen los alumnos, no los profesores. El profesor puede dar alguna consigna básica, como el objetivo general del plan de acción o los destinatarios de la campaña. Sin embargo, qué campaña realizar y cómo llevarla a cabo es decisión de los alumnos. El papel del profesor es guiar adecuadamente el proceso de elaboración, selección y planificación de la campaña con el objetivo de que sea viable y realista. Pensamos que el hecho de que sean los alumnos los que la diseñen y planifiquen puede favorecer su implicación en el proceso de aprendizaje y de implementación del proyecto.

\section{Bibliografía}

CEBRIÁN, I.; et al. (2014). Viñetas de vida. Astiberri Ediciones.

EYLER, J., y GILES, D. (1999). Where is the learning in service-learning? Jossey-Bass.

\footnotetext{
${ }^{5}$ En esta dirección se puede encontrar una plantilla de diario visual para emplear en la unidad: https://www.canva.com/design/DAC10VSO1ls/share?role=EDITOR\&token=a3rcrXEnef91SI2ROsvc9A \&utm_content $=$ DAC10VSO1ls\&utm_campaign=designshare\&utm_medium $=$ link\&utm_source $=$ sharebut ton
} 
FERrero, M. (2017). «Las ideas erróneas sobre educación». Presentado en Las pruebas de la educación, Bilbao. Recuperado el 24/4/2018, de:

https://www.youtube.com/watch?v=WchSQPiv_70

FUNDACIÓN ZERBIKAS. (2013). 60 buenas prácticas de aprendizaje servicio: inventario de experiencias educativas con finalidad social. Bilbao: Zerbikas.

INSTITUTO CERVANTES (Ed.). (2002). Marco común europeo de referencia para las lenguas: aprendizaje, enseñanza, evaluación (2. ed). Madrid: Anaya.

LuCAS, S., y MARTÍNEZ-ODRÍA, A. (2012). «La implantación y difusión del AprendizajeServicio en el contexto educativo español. Retos de futuro de una metodología de enseñanza-aprendizaje para promover la innovación en la Educación Superior». Revista del Congrés Internacional de Docència Universitària i Innovació (CIDUI), 1(1). Recuperado el 24/4/2018, de: http://www.cidui.org/revistacidui/index.php/cidui/article/view/122

Puig, J. M., BAtlle, R., Bosch, C., \& PAlos, J. (2007). Aprendizaje servicio: educar para la ciudadanía. Barcelona: Octaedro, p.53.

RodríGuez Gallego, M. R. (2014). «El Aprendizaje-Servicio como estrategia metodológica en la Universidad». Revista Complutense de Educación, 25(1). Recuperado el 24/4/2018, de: https://doi.org/10.5209/rev_RCED.2014.v25.n1.41157

Sigmon, R. (1999). An organizational journey to service-learning. En T. Stanton, J. Giles, y N. Cruz (Eds.), Service-Learning: A Movement's Pioneers Reflect on Its Origins, Practice, and Future (pp. 249-257). San Francisco: Jossey-Bass. 